\title{
Nonsurgical Management of the Boutonniere Deformity
}

\author{
Nancy S. Palchik, Douglas M. Mitchell, Naomi L. Gilbert, Amy J. Schulz, \\ Robert F. Dedrick, and Thomas D. Palella
}

The present study examined the effectiveness of a 6-week experimental program of splinting and joint protection education in reducing the progression of the early boutonniere deformity. Nine patients with mild or moderately reducible boutonniere deformities were randomized into experimental and control groups and followed for 1 year. Improvements in active extension of the proximal interphalangial joint for patients in the experimental program suggest that early initiation of a nonsurgical intervention can reduce or reverse progression of the boutonniere deformity in some patients with rheumatoid arthritis. Although the number of patients was small, we conclude that early splinting and joint protection education may limit the anatomical and functional derangements of boutonniere deformity in the rheumatoid hand.

The boutonniere deformity occurs in $15 \%-36 \%$ of all rheumatoid arthritis (RA) patients with hand involvement $[1,2]$. Despite its prevalence, there is

\footnotetext{
Portions of this paper were presented at the 1987 annual meeting of the Arthritis Health Professions Association, Washington, DC.

Nancy S. Palchik, PhD, is an Assistant Professor from the Department of Postgraduate Medicine and Health Professions Education; Douglas M. Mitchell, MS, OTR, Naomi L. Gilbert, MPH, OTR, and Amy J. Schulz, MPH, were formerly with the Department of Internal Medicine; Robert F. Dedrick, PhD, is a Research Associate from the Department of Postgraduate Medicine and Health Professions Education; and 'Thomas D. Palella, MD, is an Associate Professor from Department of Internal Medicine, Chief of the Division of Rheumatology, and Director of the Rackham Arthritis Research Unit and Michigan Multipurpose Arthritis and Musculoskeletal Diseases Center, University of Michigan, Ann Arbor, Michigan.

Address correspondence and reprint requests to Nancy S. Palchik, PhD, Department of Postgraduate Medicine and Health Professions Education, The University of Michigan Medical School, G1116 Towsley Center, Ann Arbor, MI 48109-0201.

1990 by the Arthritis Health Professions Association.
}

little information relating the deformity to the history or severity of underlying disease and little describing clinical factors associated with its progression. Management of the boutonniere deformity of rheumatoid origin has been almost entirely limited to surgical interventions. The deformity has been difficult to treat nonsurgically, in part because of the stretching of the supporting fibers, which takes place as the lateral bands displace [3] (Figure 1).

Although management of the early boutonniere deformity of traumatic origin has included splinting [4-7], there has been little research examining this intervention for deformities of rheumatoid origin and no controlled studies in either group. However, some informal case reports have indicated that splinting may be an effective intervention when initiated in the early stages of the boutonniere deformity [3, 8], and a variety of splinting interventions used to prevent and manage the deformity of rheumatoid origin have been described [9-15]. While these reports suggest that splinting a passively correctable boutonniere deformity has the potential to restore active range of motion (ROM), this has not been tested formally.

The purpose of this study was to explore the efficacy of a nonsurgical approach to managing the reducible boutonniere deformity. The specific objective of the study was to obtain preliminary information about the progression of the boutonniere deformity and the impact of a splinting and educational intervention on this progression.

\section{METHODS}

\section{Obtaining the Patient Sample}

Over a 10-month period, patients attending a university-based arthritis outpatient clinic were screened for the boutonniere deformity using a five-item symp- 


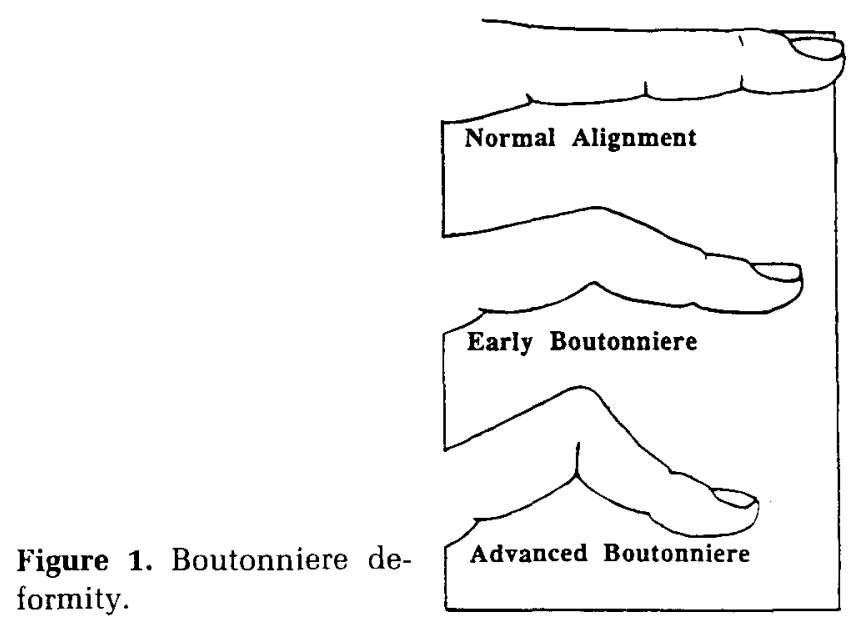

tom checklist completed by clinic physicians. The screening instrument recorded the presence or absence of arthritis or inflammation, proximal interphalangial (PIP) joint involvement, loss of ROM of the PIP joint, and the boutonniere deformity. Of 1580 patients screened, $366(23.2 \%)$ were identified with PIP involvement, $166(10.5 \%)$ were identified with loss of ROM, and $93(5.9 \%)$ were identified with a boutonniere deformity. Of the 375 patients screened with RA, $246(65.6 \%)$ had PIP involvement, 126 $(33.6 \%)$ had loss of ROM, and $73(19.5 \%)$ were identified with a boutonniere deformity.

Of the 93 patients identified through screening procedures as having a boutonniere deformity, 72 were evaluated by an occupational therapist and 56 had their boutonniere deformities confirmed. Of these patients, $21(37.5 \%)$ had reducible deformities (that is, loss of active ROM, with the joint passively moveable to full PIP extension) and $35(62.5 \%)$ had fixed deformities (that is, deformities neither reducible actively by patient nor passively by clinician).

\section{Patients}

Nine of the 21 patients with reducible deformities met eligibility criteria and agreed to participate in the splinting intervention study. Willingness to participate in the study was influenced strongly by geographic proximity to the clinic setting during the study period. Inclusion criteria were (a) boutonniere deformity of rheumatoid origin, completely passively correctable and with a loss of active PIP extension no greater than $25^{\circ}$, and (b) American Rheumatism Association anatomical stage 1,2 , or 3 [16] and functional capacity class 1, 2, or 3 [17]. Criteria for exclusion were (a) distal interphalangial (DIP) joint involvement, (b) metacarpophalangeal (MCP) subluxation, (c) peripheral neuropathy with significant sensory loss of the involved finger, or (d) any surgery of the involved finger.

Patients were randomized into experimental (splinting intervention, $n=4)$ and control $(n=5)$ groups. Characteristics of patients in both groups are shown in Table 1. Three patients in the experimental group had reducible boutonniere deformities on two fingers; one finger with a boutonniere deformity was randornly selected for the splinting intervention. The fourth patient in the experimental group had boutonniere deformities on the third, fourth, and fifth fingers of both hands; this patient was unable to follow the protocol for splint wear because of pain and inflammation of the hands and fingers, and discontinued participation early in the study. Two patients in the control group had reducible boutonniere deformities on two fingers; one finger was randomly selected for the study. The remaining three control group patients had a single reducible boutonniere deformity. Data were collected and analyzed for the three patients remaining in the experimental group and the five patients in the control group.

\section{Procedures}

Splinting Protocol. For each patient in the experimental group, the boutonniere finger was fit with a static padded gutter splint applied over the dorsal surface of the PIP joint. The splinting intervention consisted of 6 weeks of complete immobilization. Patients were instructed to wear the splint for $24 \mathrm{hr}$ a day, removing it only for personal hygiene. An individualized joint protection education program was carried out at the initial splinting session and reviewed at follow-up visits. Information was included on the rationale for splinting, correct application and precautions, and hand use following splint removal.

Outcome Measures. Patients in both the experimentall and control groups were followed for 1 year, with assessments at entry, at the completion of the 6-week splinting intervention, and at 3,6 , and 12 months. One patient in the experimental group entered the study late and was assessed at entry, 6 weeks, 3 months, and at the end of the study ( 7 months).

The boutonniere finger evaluation included measures of active and passive ROM (extension/flexion) using a finger goniometer for the MCP joint, the PIP joint with the MCP joint in the neutral position, the PIP joint with the MCP joint in the midrange position, the DIP joint with the PIP joint extended, and the DIP joint with the PIP joint flexed. Because of the small number of patients, the efficacy of the splinting and educational intervention was examined using de- 
TABLE 1

Characteristics of Experimental and Control Group Patients

\begin{tabular}{|c|c|c|c|c|c|c|c|c|c|}
\hline Ref no. & Age/sex & $\mathbf{D x}$ & $\begin{array}{c}\text { Duration } \\
\text { (years) } \\
\text { of Dx }\end{array}$ & $\begin{array}{l}\text { Anat } \\
\text { stage }\end{array}$ & $\begin{array}{l}\text { Funct } \\
\text { class }\end{array}$ & $\begin{array}{l}\text { BD study } \\
\text { hand/finger }\end{array}$ & $\begin{array}{c}\text { Joint space } \\
\text { narrowing, PIP }\end{array}$ & $\begin{array}{c}\text { Other BD } \\
\text { hand/finger }\end{array}$ & $\begin{array}{c}\text { Dominant } \\
\text { hand }\end{array}$ \\
\hline \multicolumn{10}{|c|}{ Experimental group } \\
\hline 1 & $45 / F$ & RA & 6 & 2 & 2 & $\mathrm{~L} / 4$ & 1 & $\mathrm{~L} / 5$ & $\mathrm{R}$ \\
\hline 2 & $63 / \mathrm{M}$ & RA & 5 & 1 & 2 & $\mathrm{R} / 4$ & 1 & $\mathrm{~L} / 4$ & $\mathrm{~L}$ \\
\hline 3 & $27 / F$ & RA & 0.5 & 1 & 2 & $\mathrm{~L} / 5$ & 2 & $\mathrm{~L} / 4$ & $\mathrm{~L}$ \\
\hline $9^{b}$ & $30 / \mathrm{M}$ & $\mathrm{RA}$ & 8 & 1 & 2 & NA & 2 & $\begin{array}{l}\mathrm{L} / 3,4,5 \\
\mathrm{R} / 3,4,5\end{array}$ & $\mathrm{R}$ \\
\hline \multicolumn{10}{|c|}{ Control group } \\
\hline 4 & $53 / \mathrm{F}$ & RA & 6 & 2 & 2 & $\mathrm{R} / 3$ & 1 & $\mathrm{R} / 4$ & $\mathrm{R}$ \\
\hline 5 & $45 / F$ & RA & 25 & 2 & 3 & $\mathrm{~L} / 4$ & 1 & None & $\mathrm{L}$ \\
\hline 6 & $72 / \mathrm{F}$ & RA & 6 & 2 & 2 & $\mathrm{~L} / 4$ & 1 & None & $\mathrm{R}$ \\
\hline 7 & $30 / \mathrm{M}$ & $\begin{array}{l}\text { Psoriatic } \\
\text { arthritis }\end{array}$ & 1 & NA & 2 & $\mathrm{R} / 2$ & - & None & $\mathbf{R}$ \\
\hline 8 & $66 / F$ & Arteritis & 1.4 & NA & 2 & $\mathrm{~L} / 4$ & - & $\mathrm{R} / 4$ & $\mathrm{R}$ \\
\hline
\end{tabular}

a Joint space narrowing of PIP joint was assessed by radiograph at entry: $1=\frac{1}{3}$ narrowing, $2=\frac{2}{3}$ narrowing, and $3=$ obliteration of joint space.

"Patient discontinued participation early in the study and was not included in subsequent analyses.

scriptive and graphical analyses of the outcome measures.

\section{RESULTS}

\section{6-Week Evaluation}

Changes in active extension of the PIP joint over the 12 -month study period are shown in Table 2. At the completion of the 6-week splinting intervention, the three patients in the experimental group showed no evidence of the boutonniere deformity of the splinted finger as assessed by active extension of the PIP joint. Over this same time period, patients in the control group showed no improvement in active PIP extension (mean scores of $14^{\circ}$ and $16^{\circ}$ at entry and 6 weeks, respectively). Passive extension of the PIP joint remained stable at $0^{\circ}$ (full extension) for patients in both groups. Experimental group patients reported no adverse effects of splint wear and no problems adhering to the splinting protocol. They did show, however, some reduction in both active and passive flexion of the boutonniere finger.

\section{2-Month Evaluation}

All three patients in the experimental group showed signs of recurrence of the boutonniere deformity after splint removal. These signs were noted for patient 3 in month 3 and for patients 1 and 2 in month 6 of the study (Table 2). Measures of active PIP extension showed a return to baseline for patient 2 at 12 months. Recurrences were only partial for patients 1 and 3 . At the time of recurrence, patient 1 began an intermittent splinting protocol that consisted of $8 \mathrm{hr}$ of splint wear (primarily during sleeping hours) every other day for the remainder of the study.This patient showed no evidence of the boutonniere deformity at the end of the study. Four of the five patients in the control group showed either no change in their boutonniere deformity or a progression of the deformity over the study period. The fifth patient (no. 7) in the control group had psoriatic arthritis and showed no sign of the boutonniere deformity at the end of the study.

As shown in Table 2, passive PIP extension remained stable at $0^{\circ}$ throughout the study for patients in the experimental group. In the control group, four patients showed no loss of passive ROM, while the fifth patient (no. 6) lost some passive extension ability. This loss reflects a progression of the boutonniere deformity from reversible to fixed for this individual.

Measures of active flexion of the PIP joint showed a gradual return of ROM for patients in the experimental group. Flexion of the PIP joint in control group patients remained relatively stable over the study period $\left(\right.$ mean $=90^{\circ}$ both at entry and at 12 months).

\section{Comparisons of the Splinted and Unsplinted Boutonniere Deformities in Experimental Group Patients}

To explore further the effects of splinting on the boutonniere deformity, we examined the splinted and unsplinted fingers for the three experimental group patients who had boutonniere deformities on two 


\section{T'ABLE 2}

Outcome Measures for Experimental and Control Patients

\begin{tabular}{|c|c|c|c|c|c|c|c|c|c|}
\hline \multirow{2}{*}{$\begin{array}{l}\text { Ref } \\
\text { no. }\end{array}$} & \multicolumn{3}{|c|}{$\begin{array}{c}\text { Loss of active PIP } \\
\text { extension (degrees) }\end{array}$} & \multicolumn{3}{|c|}{$\begin{array}{l}\text { Loss of passive PIP } \\
\text { extension (degrees) }\end{array}$} & \multicolumn{3}{|c|}{$\begin{array}{l}\text { Active PIP flexion } \\
\text { (degrees) }\end{array}$} \\
\hline & Entry & 6 wk & $12 \mathrm{mo}$ & Entry & $6 \mathrm{wk}$ & $12 \mathrm{mo}$ & Entry & $6 \mathrm{wk}$ & $12 \mathrm{mo}$ \\
\hline \multicolumn{10}{|c|}{ Experimental group } \\
\hline 1 & 8 & 0 & 0 & 0 & 0 & 0 & 90 & 30 & 80 \\
\hline 2 & 20 & 0 & 15 & 0 & 0 & 0 & 95 & 55 & 95 \\
\hline 3 & 15 & 0 & $4^{a}$ & 0 & 0 & 0 & 90 & 60 & 90 \\
\hline \multicolumn{10}{|c|}{ Control group } \\
\hline 4 & 10 & 10 & 10 & 2 & 0 & 0 & 80 & 80 & 90 \\
\hline 5 & 15 & 15 & 20 & 0 & 0 & 0 & 95 & 90 & 95 \\
\hline 6 & 15 & 18 & 20 & 0 & 0 & 10 & 90 & 90 & 85 \\
\hline 7 & 7 & 10 & 0 & 0 & 0 & 0 & 95 & 95 & 95 \\
\hline 8 & 25 & 25 & 25 & 0 & 0 & 0 & 95 & 95 & 90 \\
\hline
\end{tabular}

${ }^{a}$ Final assessment of patient 3 was carried out at 7 months.

fingers (Table 1). Results are shown in the figure for active and passive extension of the unsplinted finger and for active extension of the splinted finger. Passive extension of the splinted finger remained stable at $0^{\circ}$ for all three patients, and is not shown in the figure.

When splinted and unsplinted fingers were compared, the three experimental group patients showed different patterns of PIP joint extension. Patient 1 showed little evidence of the boutonniere deformity for either the splinted or unsplinted finger at the end of the study. In contrast, patient 2 experienced a recurrence of the boutonniere deformity in the splinted finger after 3 months and, at the end of the study, showed no difference between splinted and unsplinted fingers in ability to extend the PIP joint actively. There were, however, considerable differences between fingers in passive extension of the PIP joint. Passive extension for the splinted finger remained stable at $0^{\circ}$ throughout the study, while passive extension for the unsplinted finger progressed from $2^{\circ}$ at entry to a $15^{\circ}$ fixed deformity at 12 months.

Although patient 3 had some recurrence of the boutonniere deformity in the splinted finger following the 6-week intervention, the patient continued to show differences between the splinted and unsplinted boutonniere fingers for both active and passive extension of the PIP joint. Loss of active PIP extension for the splinted finger was considerably less than for the unsplinted finger $\left(4^{\circ}\right.$ and $25^{\circ}$, respectively, at the completion of the study); the unsplinted finger showed little change over the course of the study. Although passive extension of the splinted finger remained stable at $0^{\circ}$ throughout the study, passive extension of this patient's unsplinted finger progressed from $0^{\circ}$ at entry to $15^{\circ}$ at the completion of the study.

\section{DISCUSSION}

Although the number of patients was small, our results suggest that early initiation of a nonsurgical intervention may reduce or reverse the progression of the boutonniere deformity in some patients with RA. The 6-week continuous splinting intervention appeared to be effective in reducing the boutonniere deformity in the three experimental group patients as indicated by improvements in active extension of the PIP joint. Patients in the control group showed no improvements in their boutonniere deformities over this same time period. Although patients in the experimental group showed some reduction in active flexion of the boutonniere finger following removal of the splint, ability to flex the PIP joint gradually returned for these patients.

All three patients in the experimental group showed some signs of recurrence of the boutonniere deformity after removal of the splint. One patient experienced a return to baseline in ability to extend the PIP joint actively. For the remaining two patients, recurrence of the boutonniere deformity was only partial. Both of these patients remained well below the control group mean for active extension of the PIP joint.

Of particular interest in this study were compari- 

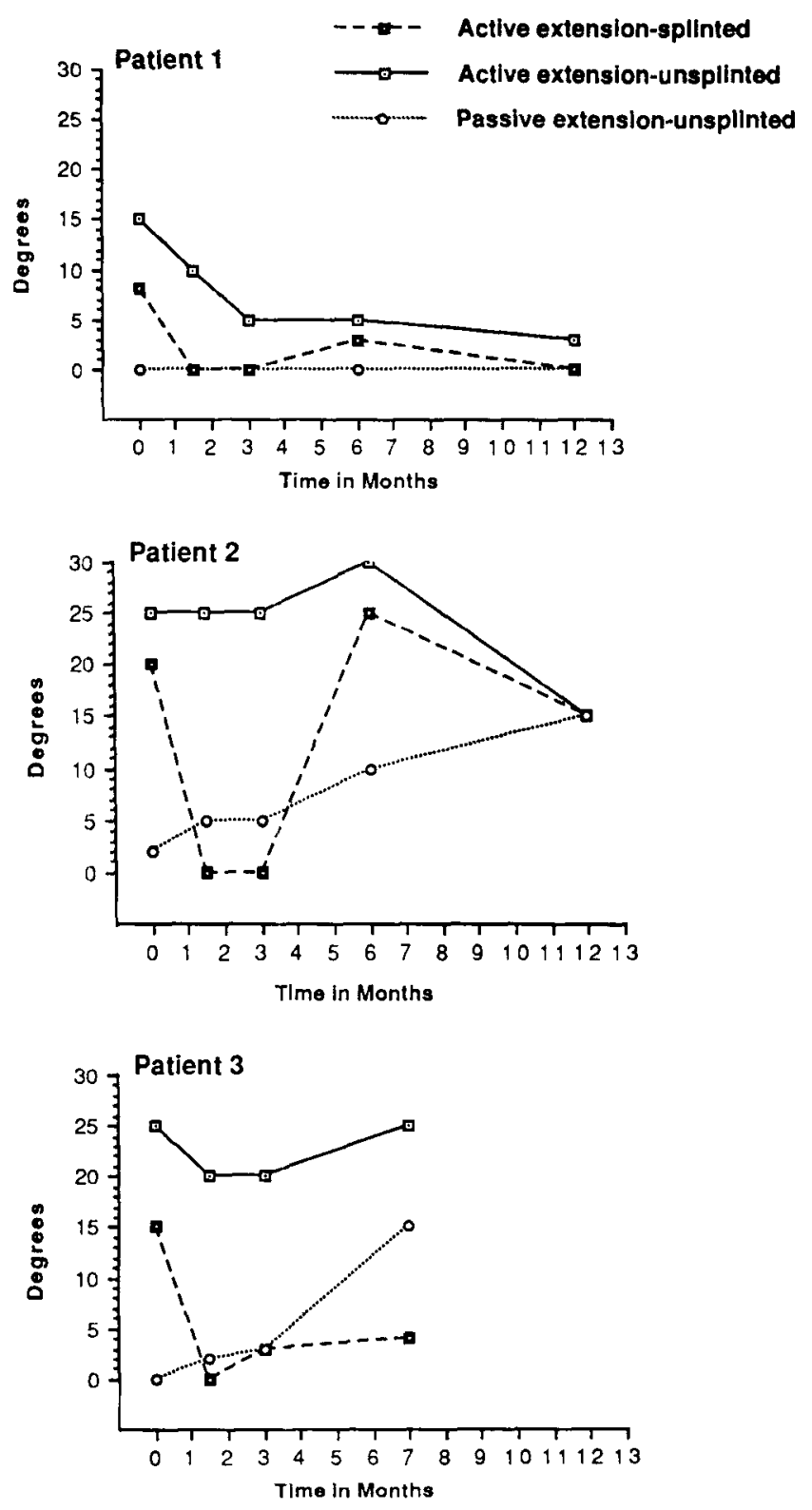

Figure 2. Extension of the proximal interphalangial joint for splinted and unsplinted fingers of experimental group patients.

sons of the splinted and unsplinted boutonniere fingers for patients in the experimental group (Figure 2). For two of the three experimental group patients, passive extension of the PIP joint of the unsplinted boutonniere finger progressed to a $15^{\circ}$ fixed deformity, while passive extension of the PIP joint of the splinted finger remained at $0^{\circ}$. These patients effectively served as their own controls, providing further support for the efficacy of the splinting intervention in limiting progression of the boutonniere deformity.
At the time of recurrence of the boutonniere deformity, one patient in the experimental group began an intermittent splinting protocol consisting of $8 \mathrm{hr}$ of splint wear every other day. This patient showed no evidence of the boutonniere deformity at the end of the study. This same patient experienced a concomitant reduction in the boutonniere deformity of the unsplinted finger. It appears likely that the proximity of the splinted and unsplinted fingers, and the location of these fingers on the nondominant hand, may have reduced mobility of the unsplinted finger and limited progression of the deformity. These results suggest that it may not be necessary to splint continuously over a 6-week period to achieve the benefits of the splinting intervention. In addition, an intermittent splinting protocol may enhance patient compliance and enable patients to continue splint use over a longer period of time. This should increase the effectiveness of the intervention, as the gradual return of the boutonniere deformity only occurred following discontinuation of splint use.

The results of the initial screening program to detect individuals with PIP involvement and with boutonniere deformities support the findings of previous investigators, suggesting that individuals with RA and PIP involvement are at relatively high risk for developing boutonniere deformities. Of the 375 patients identified with RA, almost $66 \%$ had PIP involvement. The prevalence of PIP involvement and the boutonniere deformity among arthritis outpatient clinic patients with RA, as well as the relative simplicity of the screening procedures developed for this study, suggest that screening patients at risk for developing the boutonniere deformity may be one feasible way to identify patients with the early deformity in order to initiate early intervention procedures. Most patients with a boutonniere deformity screened in the present study had a fixed deformity. This finding underscores the importance of early detection and the need for further research to test intervention strategies for decreasing progression of the deformity from its potentially reducible to fixed stage.

The results of this study provide descriptive data about the progression of the boutonniere deformity in patients with early, reducible deformities. Although further studies using a larger number of cases are needed, our preliminary results suggest that early splinting and joint protection education may be effective in limiting the anatomical and functional derangements of boutonniere deformity in the rheumatoid hand.

This work was supported in part by Multipurpose Arthritis and Musculoskeletal Diseases Center grant 2P60 AM 20557, National 
Institute of Arthritis and Musculoskeletal and Skin Diseases. The authors thank Dean S. Lewis, MD, for his valuable suggestions throughout the project; Leslie H. Kamil, MS, JD, Beverly Jacober, MS, OTR, and Linda K. Schroeder, LPT, for their assistance in the testing of the splinting intervention; and Rose Myers for her assistance in the preparation of the manuscript.

\section{REFERENCES}

1. Canosos JJ: Articular structures: Hands, wrists, and elbows. In Cohen AS, Bennett JC (eds): Rheumatology and Immunology, 2nd ed. New York, Harcourt Brace Jovanovich, 1986

2. Haldeman J, Robinson HS: Swan neck and other hand deformities in patients with RA. Physiother Can 34:13-18, 1982

3. Melvin JL: Rheumatic Disease: Occupational Therapy and Rehabilitation, 2nd ed. Philadelphia, FA Davis, 1982

4. Souter WA: The problem of boutonniere deformity. Clin Orthop 104:116-133, 1974

5. Boyes JH: Bunnell's Surgery of the Hand, 5th ed. Philadelphia, Lippincott, 1970

6. McDougall D: Modern concepts in hand orthotics. Hand 7:58-59, 1975

7. Wilson RL, Carter MS: Joint injuries in the hand: preservation of proximal interphalangeal joint function. In Hunter JM, Schneider L.H, Mackin EJ, Callahan AD (eds): Rehabilitation of the Hand, 2nd ed. St L_ouis, CV Mosby, 1984

8. Sliwa JL: Occupational therapy assessment and management. In Erlich GE (ed): Rehabilitation Management of Rheumatic Conditions, 2nd ed. Baltimore, Williams and Wilkins, 1986

9. Souter WA: Splintage in the rheumatoid hand. Hand 3:144-151, 1971

10. Seeger $M$ : The roles of splinting and rest. In Bluestone R (ed): Rheumatology. Boston, Houghton Mifflin, 1980

11. Evans DM: The PIP joint. Clin Rheum Dis 10:631-656, 1985

12. Bennett R: Orthotic devices to prevent deformities of the hand in rheumatoid arthritis. Arthritis Rheum 8:1006-1018, 1965

13. Koch R, Bird D: Orhthoses for rheumatoid fingers. Orthot Prosthet $34: 25-32,1980$

14. Gerber LH: Rehabilitation of patients with rheumatic diseases. In Kelley WM, Harris ED, Ruddy S, Sledge CB (eds): Textbook of Rheumatology, 2nd. Philadelphia, WB Saunders, 1985

15. Millender LH, Nalebuff EA: Evaluation of early rheumatoid hand involvement. Orthop Clin North Am 6:837-860, 1975

16. Steinbrocker O, Traeger CG, Batterman RC: Therapeutic criteria in rheumatoid arthritis. JAMA 140: 659-662, 1949

17. Robinson WD, Boland EW, Bunim JJ, et al.: Tenth rheumatism review. Ann Intern Med 39:498-618, 1953 